\title{
La accesibilidad web de las bibliotecas públicas del Estado frente a las entidades bancarias del Banco de España
}

\author{
Comparing the web accessibility of Spanish public libraries against Spanish financial institutions
}

\section{Beatriz Bermejo-Pantoja (1), Cristina Faba-Pérez (2)}

(1) Archivo. Arzobispado Mérida-Badajoz, C/ Obispo San Juan Rivera, 13, 06002 Badajoz (España). E-mail: beaberpan@gmail.com. (2) Facultad de Ciencias de la Documentación y la Comunicación, Universidad de Extremadura. Plaza Ibn Marwan, La Alcazaba, 06071 Badajoz, España. E-mail: cfabper@unex.es

\begin{abstract}
Resumen
Este trabajo compara la accesibilidad web de dos tipos de instituciones con características muy diferentes: las bibliotecas públicas del Estado de España y los bancos registrados en el Banco de España con establecimiento en el país, con el fin de comprobar cuál de las dos tipologías resulta más accesible para la sociedad en general. Para ello, se emplea la herramienta TAW (Test de Accesibilidad Web) que utiliza las guías de accesibilidad Web Content Accesibility Guidelines (WCAG) 2.0. Como principal resultado se obtiene que las bibliotecas públicas del Estado cuentan con un promedio de errores de accesibilidad menor que los bancos, siendo la pauta menos vulnerada en ambos conjuntos la 1.2 que presenta alternativas para la percepción del audio/vídeo, subtítulos y audio-descripciones del contenido para los casos en los que los ciudadanos padezcan problemas de audición y/o visión. Se concluye con la necesidad de diseñar sitios web más accesibles para todos los ciudadanos, tanto por parte de instituciones con intereses sociales, como empresariales.
\end{abstract}

Palabras clave: Accesibilidad web. Bibliotecas Públicas del Estado. Banca. Análisis comparativo. España.

\section{Introducción}

En la actualidad, las bibliotecas públicas constituyen un espacio cívico del que toda la comunidad puede disfrutar sin ningún coste añadido. Los profesionales que trabajan en estos servicios tienen la misión de "relacionar a los vecinos, reavivar el compromiso cívico, impulsar la participación ciudadana y fomentar el mayor grado de intervención en los procesos de decisión y solución de los problemas de la comunidad" (Ford, 2002). En la evolución de esta nueva perspectiva social que muestra la biblioteca pública, López López (2007) reproduce las palabras de la International Federation of Library Associations and Institutions (IFLA, 2002) al señalar que las bibliotecas públicas juegan un papel importante

\begin{abstract}
This article compares the web accessibility of two types of institutions with different characteristics: the public libraries of the State of Spain and the banks registered in the Bank of Spain with establishment in the country. The objective of this investigation is to verify which of the two types turns out to be more accessible for the community. For it, the tool TAW (Test of web Accessibility) was used, based on the "Web Content Accessibility Guidelines (WCAG) 2.0". The principal result is that the public libraries have a lower average of accessibility errors than banks. The best guideline in both populations is 1.2. This guide presents alternatives for the perception of the audio/ video, subtitles and audio content description for citizens with hearing or vision problems. It is concluded that it is necessary to design more accessible web sites for all citizens, both by institutions with social interests, and for business purposes.
\end{abstract}

Keywords: Web accessibility. State public libraries. Bank entities. Comparative analysis. Spain.

en los valores democráticos y en la participación ciudadana de todos los colectivos sociales.

Al contrario que las bibliotecas públicas, las entidades bancarias son "empresas" que trabajan para captar clientes y ofrecerles sus servicios por intereses económicos. La banca ha superado sus límites dando lugar al concepto de banca ética. Este concepto en España aún no tiene mucha repercusión, pues consiste en ofrecer los servicios propios de la banca, pero con fines éticos. Este paradigma puede desencadenar dos perspectivas: aquellos productos denominados éticos por los que la entidad bancaria adquiere un beneficio monetario; o aquellos cuya cuantía recaudada se aplica a un compromiso ético y social por parte de la entidad bancaria (San Emeterio y Retolaza, 2003), en tal caso, la 
entidad bancaria también adquiere beneficios en el marketing de imagen, pero de forma indirecta.

Al igual que la finalidad de las bibliotecas públicas (perspectiva social) y de las entidades bancarias (perspectiva económica) son dispares, también lo son los intereses de sus destinatarios y los servicios y productos que suministran. Así, el objetivo principal de la biblioteca pública es ofrecer "servicios" para que todas las personas, ya sean "usuarios" reales o potenciales, puedan utilizarlos (préstamo, sala de lectura, talleres divulgativos-creativos, etc.) independientemente de su edad, raza, sexo, religión, nacionalidad, idioma o condición social (IFLA, 1994), mientras que la banca, aunque también suministra servicios, tiene como objetivo fundamental "vender" un "producto" al "cliente" (fondos de inversiones, fondos de pensiones, tarjetas de crédito-débito...) creándole una necesidad.

Hoy en día, la mayoría de las bibliotecas públicas y de las entidades bancarias disponen de sitios webs para exponer su información y acceder a sus servicios. Sin embargo, es preciso analizar el grado de accesibilidad de ambos conjuntos para conocer el grado de penetración en la sociedad.

\subsection{Objetivos e hipótesis}

El objetivo principal de este trabajo es comparar la accesibilidad web de dos tipologías de instituciones ubicadas en la misma zona geográfica (España), las bibliotecas públicas del Estado y los bancos registrados en el Banco de España con establecimiento en el país, con una idiosincrasia muy diferente en cuanto a su finalidad, servicios/productos y destinatarios, con el fin de comprobar cuál de las dos tipologías resulta más accesible para la sociedad en general.

La hipótesis de partida es que si las bibliotecas públicas estatales son instituciones con vocación de servicio a la sociedad, mientras que la principal finalidad de las entidades bancarias es económica, el grado de accesibilidad web de los bancos debería ser superior o, como mínimo, igual al de las bibliotecas públicas, a fin de favorecer el acceso a los mismos y, con ello, su incremento económico.

El trabajo se justifica porque, aunque hay investigaciones que estudian la accesibilidad de las bibliotecas públicas (Lilly y Van Fleet, 2000; López Marín, Méndez Rodríguez y Sorli Rojo, 2002; Brobst, 2009; Conway, 2011; Park, 2012; Maatta Smith, 2014, etc.) o de entidades bancarias (Pulido Granados y Medina García, 2008; Fernández Cortés, 2009; Khan, Mahapatra y Sreekumar, 2009; Martínez, Andrés y García, 2014, etc.) no hemos localizado, hasta el momento, ninguna investigación que compare la accesibilidad de dos conjuntos tan opuestos.

\subsection{Las bibliotecas públicas y la tecnología}

Aunque existen muchas definiciones de biblioteca pública, en este caso, vamos a limitarnos a la establecida en 2001 por la IFLA, quien la define como:

Una organización establecida, respaldada y financiada por la comunidad, ya sea por conducto de una autoridad u órgano local, regional o nacional, o mediante cualquier otra forma de organización colectiva. Brinda acceso al conocimiento, la información y las obras de imaginación gracias a toda una serie de recursos y servicio y está a disposición de todos los miembros de la comunidad por igual, sean cuales fueran su raza, nacionalidad, edad, sexo, religión, idioma, discapacidad, condición económica y laboral y nivel de instrucción.

Dentro de las bibliotecas públicas, se encuentran las estatales. España cuenta con 53 bibliotecas públicas estatales, presentes en las diferentes comunidades autónomas con el fin de favorecer el desarrollo cultural de país y el acceso a la información de los ciudadanos (Ministerio de Educación, Cultura y Deporte, 2018).

Con respecto a las bibliotecas públicas del Estado, el trabajo de Jaime L. Peón (1991) define sus principales funciones: reunión, organización y servicio público de las colecciones formadas por los materiales bibliográficos, gráficos y audiovisuales; promoción de los materiales nombrados anteriormente, así como su utilización, conservación y el enriquecimiento del patrimonio bibliográfico; recepción, en las capitales de provincia, de al menos un ejemplar de las obras del Depósito Legal; y cooperación con las demás Bibliotecas Públicas del Estado y con las demás de su comunidad autónoma.

Como se acaba de señalar, tanto en la definición de biblioteca pública, como en el concepto de biblioteca pública del Estado, todos los colectivos sociales deben tener acceso a la biblioteca, física y digital, en igualdad de condiciones.

Con respecto a la biblioteca digital, debido a la constante evolución de las nuevas tecnologías, las bibliotecas se han visto obligadas a mejorar y adaptarse al nuevo desarrollo tecnológico con el fin de permanecer activas. Este gran cambio que ha revolucionado el día a día de las bibliotecas ha afectado a los bibliotecarios, obligados a formarse en el nuevo ámbito tecnológico para proporcionar a los usuarios todas las posibilidades que, gracias a las nuevas tecnologías, pueden ofrecer los servicios de la biblioteca (DelmásRuiz y López-Borrul, 2015). 
Como señalan Sandra Sánchez y Santiago Yubero (2015), al igual que los bibliotecarios, los usuarios también tienen que ser sometidos a procesos de alfabetización digital, ya que las tecnologías de la información han replanteado la forma de acceder a la información y el conocimiento, además de la comunicación y la participación ciudadana.

\subsection{La banca y la tecnología}

En España, la institución responsable de regular las entidades bancarias es el Banco de España, que remonta su origen al siglo XVIII, creado por la Real Cédula de Rey Carlos III. Actualmente, es el Banco Central Nacional y supervisor del sistema bancario español junto al Banco Central Europeo y se regula mediante la Ley de Autonomía del Banco de España. Como miembro del Sistema Europeo de Bancos Centrales (SEBC), el Banco de España desarrolla las siguientes funciones (Banco de España, 2018), que difieren según el papel que represente: definir y establecer la política monetaria en la zona euro; llevar a cabo las operaciones de cambio de divisas; promover el buen funcionamiento de los sistemas de pago en la zona euro; y emitir billetes.

El Banco de España cuenta con un Registro de Entidades, donde se especifican todos sus datos identificativos, que son supervisadas por el Banco de España y por el Banco Central Europeo en el marco del Mecanismo Único de Supervisión, como las entidades de crédito extrajeras y filiales de entidades de crédito extranjeras autorizadas para operar en España sin establecimiento en el país (Banco de España, 2018).

Mediante los avances que se han experimentado con las nuevas tecnologías, la tradicional banca ha abierto sus puertas al mundo digital. Actualmente, Internet brinda ventajas como la mejora en el alcance y el coste de los servicios, así como el acceso, independientemente del horario de apertura (Hernández Ortega, Jiménez y Martín, 2010). La banca online es una de las herramientas que proporcionan los bancos tradicionales a los clientes (llegando en ocasiones a ser sustituidos por ellas). Algunas de las ventajas que ofrecen los bancos online son: disponibilidad horaria 24h/7días a la semana; transparencia de la web a la hora de proporcionar información detallada; asesoramiento personalizado a través de la plataforma y contratación de productos a distancia; reducción de las comisiones debido al menor coste que ocasiona la eliminación de oficinas físicas; y amplio abanico de posibilidades que ofrece mediante la incorporación de las nuevas tecnologías (Merlo, Picart y Monforte, 2018).
El desarrollo de la banca online implica que los ciudadanos realicen algunas de las operaciones para gestionar sus ahorros a través de Internet, que puede ser un cúmulo de ventajas a la hora de realizar actividades cotidianas a través de un navegador sin tener la necesidad de desplazarse, pero también puede ser una amenaza a la seguridad y ahorros del ciudadano. El artículo de Lara y Albán (2017), sobre el carácter delictivo de las operaciones bancarias realizadas a través de Internet, indica el gran desarrollo global de las leyes que protegen este tipo de transacciones fraudulentas. Precisamente, el trabajo de López, Rodríguez y González (2010) hace hincapié en la generación de confianza y la calidad del sitio web financiero. Establece que los parámetros para analizar la calidad de un sitio web con éxito son la información que ofrece, su estructura y diseño.

\subsection{La accesibilidad web}

La accesibilidad web se aborda desde diferentes puntos de vista. Siguiendo a Carrasco de la Rica y Faba-Pérez (2017), aunque hay autores e instituciones que asocian la accesibilidad web principalmente con las personas con discapacidad (Henry, 2002; World Wide Web Consortium (W3C) http://www.w3.org/), sin embargo, debido a los cambios y la evolución tecnológica constante, otros investigadores afirman que la accesibilidad web no solo abarca a los usuarios con discapacidad sino que también engloba otros tipos de dificultades de acceso (Berners-Lee, 2000; HassanMontero y Martín-Fernández, 2005; CaballeroCortés, Faba-Pérez y Moya-Anegón, 2009), por lo que se puede señalar que la accesibilidad web permite el acceso a los sitios web a todas las personas, independientemente de si sufren discapacidad, de su entorno y de su modo de acceso.

La elaboración de una página web accesible, así como su evaluación, no es una tarea fácil (Serrano Mascaraque, 2009). Y las nuevas tecnologías, que deben suponer un avance en la accesibilidad, también puede provocar una reacción contraria si el diseño de accesibilidad no es el adecuado (López Marín, Méndez Rodríguez y Sorli Rojo, 2002).

Para la normalización de este concepto se desarrolla una normativa relacionada con la accesibilidad digital con el fin de proteger los derechos de las personas con discapacidad, además de otros derechos, como el derecho a la información, a la educación y a la sanidad (Luján Mora, 2018). Algunas de estas normativas son la norma UNE 139802:1998 EX, la Ley 34/2002 y el Real Decreto $1414 / 2006$, entre otras muchas. 


\section{Datos y metodología}

\subsection{Datos}

Las instituciones públicas que se han seleccionado para este trabajo son las bibliotecas públicas del Estado, obtenidas de la web Panorámica de las 53 Bibliotecas Públicas del Estado (http://mapabpe.mcu.es/mapabpe.cmd) del Ministerio de Educación, Cultura y Deporte del Gobierno de España (Apéndice). Aunque son 53, trabajamos con 51 porque la Biblioteca Pública del Estado de Madrid "Manuel Alvar" y la Biblioteca Pública de Vitoria-Gasteiz no pasan el test de accesibilidad (1).

En cuanto a las instituciones bancarias, se ha utilizado el listado de bancos resultante de la Consulta de datos actuales de entidades registradas en el Banco de España, obtenido de la web del Banco de España (http://app.bde.es/ren/html/ini cio.htm) (Apéndice). Los 58 bancos originales del listado se convierten en 52, puesto que el Banco de Caja España de Inversiones, el Banco de Castilla-La Mancha, el Banco Industrial de Bilbao y el Citibank España carecen de sitio web, y el banco Santander Consumer Finance y Self Trade Bank no tienen sus webs operativas.

\subsection{Metodología}

El Test de Accesibilidad Web (TAW) (https:// www.tawdis.net/index) es la herramienta utilizada para el análisis de problemas que pueden presentar los sitios webs, tanto de las bibliotecas públicas del Estado (BPE), como de los bancos registrados en el Banco de España (BE). Esta herramienta, desarrollada por el Centro Tecnológico de la Información y de la Comunicación en España, utiliza las "Pautas de Accesibilidad al Contenido en la Web", originalmente denominadas Content Accessibility Guidelines (WCAG) 2.0 establecidas por la World Wide Web Consotium (W3C) mediante el grupo Web Accessibility Iniciative (WAI). Aunque desde junio de 2018 existe una versión más avanzada de las WCAG 2.0 (WCAG 2.1), la recolección de datos de este trabajo se realiza en los meses de abril y mayo de 2018, por lo que ha sido la versión 2.0 la utilizada en la presente investigación. Además, a fecha de marzo de 2019, TAW seguía utilizando las WCAG 2.0, que se estructuran en 4 principios de accesibilidad, 12 pautas (Tabla I) y 61 criterios de éxito, que conforman las pautas y a los que se le asigna un nivel de conformidad ( $A, A$ A y AAA). El nivel más nocivo en accesibilidad web si no se cumple es el $A$, refleja los criterios de accesibilidad que "tiene" que cumplir el sitio web. AA, es un nivel perjudicial medio, es decir, "deben" cumplirse en el sitio web para que su accesibilidad sea aceptable. Y, por último, el nivel AAA es el de menor gravedad, y se aplica a los criterios de éxito cuya accesibilidad "puede" cumplirse (2).

La proporción de criterios de éxito asignados a cada nivel se muestra a continuación:

- Perceptible: A $(40,91 \%)$, AA $(22,73 \%)$, AAA $(36,63 \%)$.

- Operable: A (45\%), AA (15\%), AAA (40\%).

- Comprensible: A $(29,41 \%)$, AA $(29,41 \%)$, AAA $(41,18 \%)$.

- Robusto: A (100\%).

\section{Principio Perceptible}

1.1. Proporcionar alternativas textuales para todo contenido no textual de modo que se pueda convertir a otros formatos que las personas necesiten, tales como textos ampliados, braille, voz, símbolos o en un lenguaje más simple.

1.2. Medios tempodependientes: proporcionar alternativas para los medios tempodependientes.

1.3. Crear contenido que pueda presentarse de diferentes formas (por ejemplo, con una disposición más simple) sin perder información o estructura.

1.4. Facilitar a los usuarios ver y oír el contenido, incluyendo la separación entre el primer plano y el fondo.

Principio Operable

2.1. Proporcionar acceso a toda la funcionalidad mediante el teclado.

2.2. Proporcionar a los usuarios el tiempo suficiente para leer y usar el contenido.

2.3. No diseñar contenido de un modo que se sepa podrían provocar ataques, espasmos o convulsiones.

2.4. Proporcionar medios para ayudar a los usuarios a navegar, encontrar contenidos y determinar dónde se encuentran.

\section{Principio Comprensible}

3.1. Hacer que los contenidos textuales resulten legibles y comprensibles.

3.2. Hacer que las páginas web aparezcan y operen de manera predecible.

3.3. Ayudar a los usuarios a evitar y corregir los errores Principio Robusto

4.1 Maximizar la compatibilidad con las aplicaciones de usuarios actuales y futuras, incluyendo las ayudas técnicas

Tabla I. Pautas WCAG 2.0

(Fuente: http://www.sidar.org/traducciones/wcag20/es/ comprender-wcag20/text-equiv.html) 
Los resultados que arroja esta herramienta son los errores, en valores absolutos, de los 61 criterios de éxito y el tipo de error que le afecta: fallos, son necesarias correcciones; advertencias, se requiere revisión manual; y errores sin revisar, es imposible llevar a cabo una comprobación automática. La recolección de datos con TAW se realiza en los meses de abril y mayo de 2018 . Se generan 8 matrices, una para cada principio de accesibilidad (es decir, 4 para las BPE y 4 para los BE). En total, se analizan 18849 datos: 61 criterios de éxito $\times 3$ tipos de errores (fallos, advertencias y sin revisar) (3) x 103 entidades (51 BPE + $52 \mathrm{BE}$ ).

Los valores absolutos (Vabs) se ponen en relación con el tamaño de los sitios web en Kilobytes (KB) (hallados en Firefox Mozilla) con el fin de obtener unos resultados en valores relativos (Vrel) más representativos, que se utilizan para el cálculo del promedio de errores de accesibilidad web de cada principio, pauta, criterios de éxito y niveles de conformidad: $\mathrm{V}_{\text {rel }}=\mathrm{V}_{\text {abs }} /$ Tamaño (KB).

Con los resultados obtenidos se podrá comprobar qué población en conjunto (BPE y bancos) resulta más/menos accesible para la ciudadanía.

\section{Resultados}

\subsection{Principios}

La Figura 1 presenta la comparación del promedio de errores de accesibilidad de las BPE y los bancos registrados en el $\mathrm{BE}$ con establecimiento en nuestro país, considerando los cuatro principios de accesibilidad.

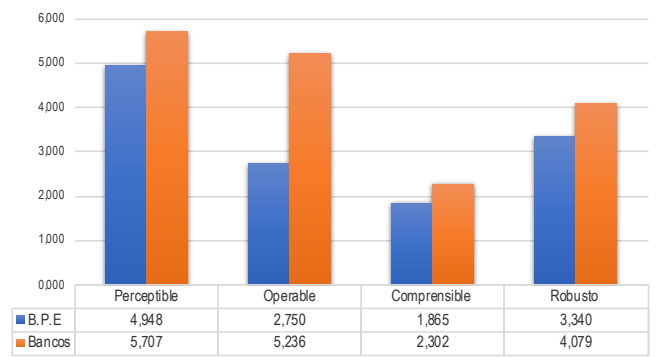

Figura 1. Principios: promedio de errores de accesibilidad web

Como se muestra, los bancos registrados en el Banco de España obtienen un promedio de errores más elevado que las bibliotecas públicas del Estado en todos los principios (sumando los promedios de todos los principios, las BPE obtienen 12,903 y los BE alcanzan 17,324). Se aprecia que el principio Perceptible es el que más errores tiene, tanto en las BPE como en los bancos registrados en el B.E, 4,948 y 5,707 respectivamente. Al contrario, el principio Comprensible es el que cuenta con un promedio de errores menor $(1,865$ en las BPE y 2,302 en los bancos registrados en el BE). Este hecho resulta positivo para ciertos colectivos sociales, como la tercera edad, ya que el principio Comprensible, por ejemplo, hace más legibles y claros los contenidos textuales de las webs o ayuda a evitar y a corregir errores (pensemos que hablamos de un colectivo cuyos cambios físicos y psicológicos afectan a su capacidad cognitiva o lectora).

En el gráfico también se observa que la diferencia de errores entre ambos parámetros es más representativa en el principio Operable, con una variación de 2,486 (5,236 errores en los bancos registrados en el B.E y 2,75 en las BPE) frente a 0,759 en el principio Perceptible (5,707 en bancos y 4,948 en BPE), 0,442 en Comprensible (2,32 en Bancos y 1,86 en BPE) y 0,739 en el principio Robusto $(4,079$ en Bancos y 3,34 en BPE).

\subsection{Pautas}

La Figura 2 compara el promedio de errores de accesibilidad entre los dos conjuntos analizados teniendo en cuenta las 12 pautas de accesibilidad. La mayoría de las pautas de las B.P.E (9 de 12) tienen un promedio de errores menor que los bancos registrados en el $\mathrm{BE}$, excepto la pauta 1.1. Texto alternativo, 1.2. Medios tempodependientes y 2.4. Navegable.

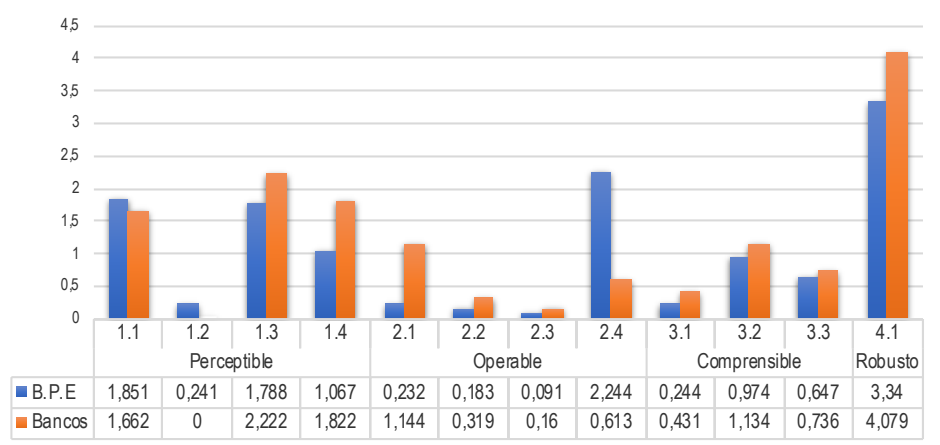

Figura 2. Pautas: promedio de errores de accesibilidad web

La pauta que presenta mayor número de errores entre los dos conjuntos estudiados es la 4.1. Compatible, del principio Robusto con un promedio de 7,419 (bibliotecas 3,34 y bancos 4,079); seguida de 1.3. Legible, del principio Perceptible, con un total de 4,01 (1,788 en BPE y 2,222 en bancos registrados en el BE). Se observa que la pauta con menos errores es la 1.2. Medios tempodependientes del principio Perceptible con un promedio de 0,241 en las BPE y 0 en los bancos registrados en el BE Para ciertos colectivos con diversidad funcional auditiva y visual, resulta muy 
positivo que la Pauta 1.2. sea la menos vulnerada, ya que presenta alternativas entre el audio y el vídeo, y subtítulos para el caso en el que el usuario/cliente padezca problemas de audición; y audiodescripción del contenido para los casos en los que presente problemas de visión.

\subsection{Criterios de éxito}

\subsubsection{Criterios de éxito del principio Perceptible}

En la Figura 3 se muestran los 22 criterios de éxito asociados a las 4 pautas del principio Perceptible. Los criterios de éxito que mayor promedio de errores obtienen aunando los resultados de ambos conjuntos son, en primer lugar, el 1.1.1. Contenido no textual, con un promedio de errores total de 3,513 (1,851 BPE y 1,662 bancos del BE); le sigue, con un número de errores también elevado, el criterio 1.3.1. Información y relaciones, con un total de 2,109. Podemos observar que 3 de los 4 criterios de éxito con más errores pertenecen al nivel de conformidad A (1.1.1., 1.3.1 y 1.3.2) aspecto negativo, ya que es el nivel esencial de accesibilidad que "tiene" que cumplir un sitio web.

Los criterios que presentan menos errores son 1.2.1-1.2.9, 1.4.2. y 1.4.7., con un promedio de errores de 0,027 , por parte de las BPE y ninguno los bancos. El 63,63\% de estos 11 criterios pertenecen al nivel AA o AAA, lo óptimo hubiera sido que fueran del nivel $A$. En cuanto a los criterios de éxito que pertenecen a la pauta 1.2., y que han obtenido el menor número de errores, ya se ha comentado en el apartado anterior (4.2) su beneficio para ciertos colectivos.

Los criterios de éxito 1.4.2. y 1.4.7. se relacionan con la regulación del sonido que pueda incluir el sitio web, subir o bajar el volumen o pausarlo, lo que también permitirá a los ciudadanos con problemas auditivos ajustarlo a sus necesidades.

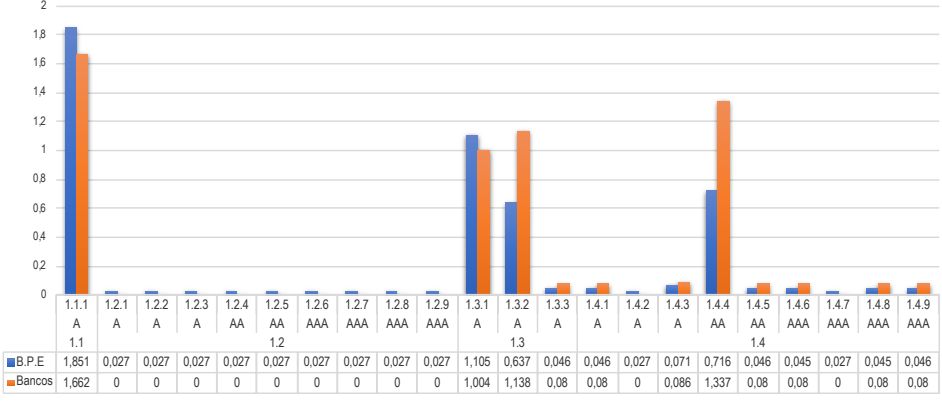

Figura 3. Criterios de éxito perceptibles: promedio de errores de accesibilidad web

\subsubsection{Criterios de éxito del principio Operable}

La Figura 4 muestra los errores de accesibilidad web de los 20 criterios de éxito asociados a las 4 pautas pertenecientes al principio Operable. Los criterios de éxito con mayor promedio de errores son: en primer lugar, 2.4.6. Encabezados y etiquetas, con un total de 1,583 (0,439 las BPE y $1,144$ los bancos registrado en el $B E)$, de nivel de conformidad AA; seguido del criterio de éxito 2.4.4. Propósito de vínculos, con un promedio de errores de 0,735 en las bibliotecas públicas del Estado y 0,747 en los bancos registrados en el Banco de España (nivel A). En este sentido, no resulta positivo que uno de los criterios con más errores sea del nivel $A$.

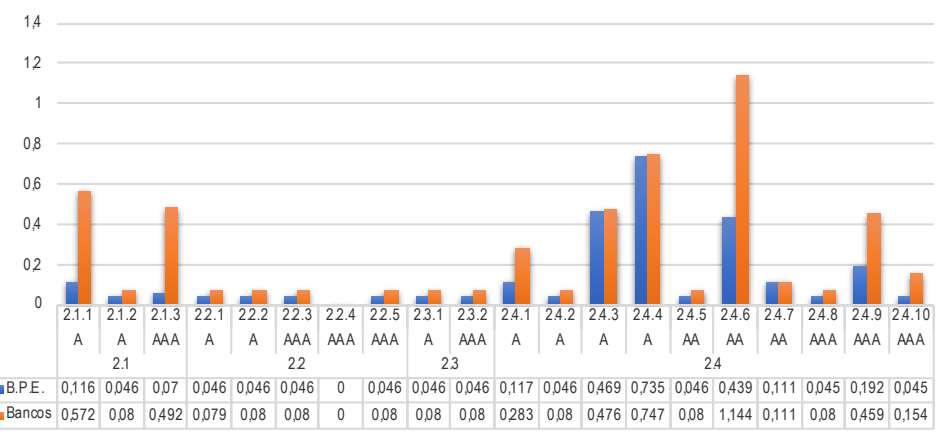

Figura 4. Criterios de éxito operables: promedio de errores de accesibilidad web

Se aprecia que el criterio de éxito con menos errores es el criterio 2.2.4. Interrupciones, con un total de 0 errores en ambos conjuntos analizados. Su nivel de conformidad es AAA. Con respecto a ciertos colectivos sociales, como las personas mayores, que no haya errores en este criterio es favorable porque les permite postergar o suprimir las interrupciones automáticas hasta que hayan tenido tiempo suficiente para leer y usar el contenido.

3.3.3. Criterios de éxito del principio Comprensible

La Figura 5 refleja el promedio de errores de los 17 criterios de éxito asociados a las 4 pautas del principio Comprensible, asociado cada uno a su correspondiente nivel de conformidad. El criterio de éxito con mayor promedio de errores con diferencia es el 3.2.5. Cambios a petición, con 0,786 en las BPE y 0,799 en bancos registrados en el B.E (total 1,585). Nunca es positivo tener errores de accesibilidad en un sitio web, pero este en concreto es de menor relevancia, ya que se presenta en un criterio de éxito con nivel de conformidad AAA. El criterio de éxito 3.3.4. Prevención de errores, es el siguiente que presenta un mayor número de errores $(0,216$ en BPE y 0,223 en 
bancos registrados en el BE) con un nivel de conformidad AA.

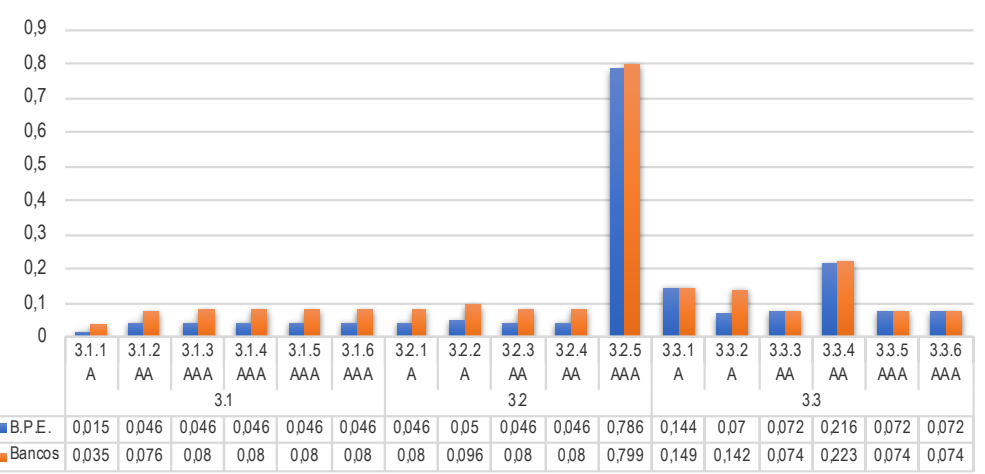

Figura 5. Criterios de éxito comprensibles: promedio de errores de accesibilidad web

El criterio con menos promedio de errores es el 3.1.1. Idioma de la página, con sólo 0,015 en las BPE y 0,035 en los bancos registrados en el BE. Es una puntualización positiva, ya que se trata de un criterio de éxito con nivel de conformidad $\mathrm{A}$. Este aspecto es favorable para todos los ciudadanos, ya que el idioma predeterminado del sitio web (como las ayudas técnicas, etc.) será legible y comprensible.

\subsubsection{Criterios de éxito del principio Robusto}

El principio Robusto solo tiene 2 criterios de éxitos y ambos están relacionados con una sola pauta de accesibilidad: la 4.1. Compatible. El promedio de errores es más elevado en el criterio de éxito 4.1.2. Nombre, función, valor, con 3,876 en las B.P.E, bastante superior a los bancos registrados en el BE, 0,204. Al contrario, el criterio de éxito 4.1.1. Procesamiento, cuenta con un promedio de errores bastante inferior al anterior $(0,219$ las BPE y $0,121 \mathrm{BE})$. Dada la variedad y el avance tecnológico, es importante para todos los colectivos sociales que los sitios web tengan un contenido compatible para ser interpretado de forma fiable por una amplia variedad de aplicaciones de usuarios presentes y futuras.

\subsection{Niveles de conformidad}

La Figura 6 representa la comparativa del promedio de errores de accesibilidad web de los colectivos analizados, según los tres niveles de conformidad: A, AA y AAA.

Se aprecia que los errores en el nivel de conformidad $\mathrm{A}$ son los más abundantes en los principios Perceptible, Robusto y Operable (en este orden), tanto en las B.P.E como en los bancos registrados en el BE. Este hecho hay que analizarlo con cautela teniendo en cuenta que en los 3 principios mencionados la mayor proporción de criterios de éxito se halla en el nivel A (véase Metodología).

Se observa que el principio Perceptible, tanto en las BPE, como en los bancos, tiene un promedio de errores muy elevado $(3,792$ y 3,964 , respectivamente); en el principio Operable, el nivel de conformidad que más promedio de errores acoge es también el A, con 1,664 en las B.P.E y 2,476 en los bancos registrados en el BE; el principio Robusto tiene sólo 2 criterios de éxito y ambos pertenecen al nivel de conformidad A, por lo que, sus errores también pertenecen a tal nivel: 3,340 errores promedio en las BPE y 4,079 en los bancos registrados en $\mathrm{BE}$.

Al contrario que los principios analizados anteriormente, el principio Comprensible tiene más promedio de errores en los criterios de éxito que pertenecen al nivel de conformidad AAA, 1,113 en las BPE y 1,267 en los bancos registrados en el BE.

Como se puede comprobar, en todos los niveles de conformidad de los cuatro principios, menos en el AAA del principio Perceptible, los bancos registrados en el Banco de España tienen mayor promedio de errores que las bibliotecas públicas del Estado.

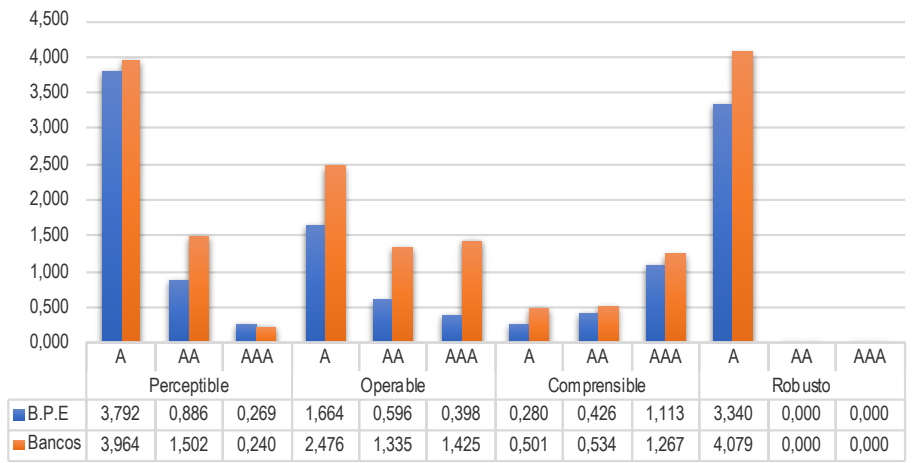

Figura 6. Niveles de conformidad: promedio de errores de accesibilidad web

\section{Conclusiones y análisis de hipótesis}

Tanto las bibliotecas públicas como las instituciones bancarias ofrecen cada vez más servicios/productos a sus usuarios/clientes a través de sus sitios web lo que, a priori, permite una mayor comodidad. Ahora bien, es preciso analizar su grado de accesibilidad web, a fin de comprobar hasta qué punto están resultando útiles para la sociedad.

En este sentido, las conclusiones a las que se ha llegado en esta investigación se muestran a continuación: 
Respecto a los Principios de accesibilidad, se destaca que las bibliotecas públicas del Estado cuentan con un promedio de errores menor que los bancos registrados en el Banco de España con establecimiento en este país. De forma conjunta, los errores más frecuentes son los que afectan a la percepción del sitio web, y los menos comunes los que influyen en su comprensión. Este hecho incide en que los sectores de la población que presentan cierta disfunción visual o auditiva no pueden percibir correctamente el contenido que se disponga en los sitios webs, pero sí pueden comprender estos contenidos e interaccionar con ellos.

En relación a las Pautas, en el total de las mismas (12), las entidades bancarias registradas en el Banco de España son más vulnerables que las bibliotecas públicas estatales. La Pauta 4.1. Compatible es la que acumula más errores, mientras que la pauta 1.2. Medios tempodependientes es la que menos errores presenta. Concluyendo, las webs tienen dificultades para maximizar su compatibilidad con diferentes aplicaciones de usuarios, pero sí disponen de alternativas como subtítulos, audiodescripciones, etc. para que el ciudadano con problemas auditivos/visuales pueda apreciar todo el contenido multimedia.

Profundizando en los Criterios de éxito, los que han obtenido resultados más beneficiosos, es decir, menor número de errores de accesibilidad, se relacionan con las alternativas entre el audio o el video y los subtítulos, las autodescripciones o la regulación del sonido -subir o bajar el volumen o pausarlo, todo ello favorable para las personas con dificultades auditivas y visuales (criterio/s del principio Perceptible); parar o suprimir las interrupciones que puedan producirse en la interacción con los sitios web, positivo para que los usuarios/clientes tengan tiempo suficiente para leer y usar el contenido (criterio del principio Operable); tener el idioma predeterminado del sitio web legible y claro (criterio del principio Comprensible); y un contenido compatible interpretable de forma fiable por una amplia variedad de aplicaciones de usuarios presentes y futuras (criterio del principio Robusto).

Por último, en cuanto a los Niveles de conformidad, las entidades bancarias registradas en el Banco de España presentan un mayor número de errores de accesibilidad de todos los niveles, en comparación con las bibliotecas públicas estatales. Ambos conjuntos dan como resultado los tres niveles de conformidad en el siguiente orden de vulnerabilidad de mayor a menor: A, AA y AAA. Este orden no resulta favorable, ya que los menos vulnerados deberían haber sido los $\mathrm{A}$, seguidos de los AA y, por último, los AAA.
Al comprobarse que las bibliotecas públicas estatales obtienen menos errores de accesibilidad web que las entidades bancarias registradas en el Banco de España en todos los principios analizados y en 9 de las 12 pautas, la hipótesis de partida no se cumple, por lo que instituciones con fines sociales, como las bibliotecas públicas, parecen estar más preocupadas en hacer accesibles sus sitios web, que instituciones con una visión más empresarial, como los bancos.

A la vista de las conclusiones obtenidas e hipótesis no corroborada, se propone realizar este análisis con las mismas poblaciones pasado un tiempo prudencial para comprobar si es posible corroborar la hipótesis planteada o, por el contrario, los resultados siguen la misma tendencia. También se plantea profundizar en el diseño de sitios web de bibliotecas públicas y entidades bancarias más accesibles para todos, llevando a cabo un estudio de necesidades de accesibilidad web a través de la evaluación heurística con diversos colectivos (personas con diversidad funcional, tercera edad, etc.).

\section{Notas}

(1) Debido a que las webs propuestas por el Ministerio de Educación, Cultura y Deporte dirigen hacia "El Portal del Lector" de todas las Bibliotecas de la Comunidad de Madrid y a la página web de la Diputación Foral de Álava, respectivamente.

(2) Los 61 criterios de éxito pueden consultarse: http://www.sidar.org/traducciones/wcag20/es/

(3) Por motivos de espacio, en los Resultados no se desglosan en fallos, advertencias y sin revisar.

\section{Referencias}

Banco de España. Sobre el Banco. https://www.bde.es/ bde/es/secciones/sobreelbanco/ (2018-06-18).

Berners-Lee, T. (2000), Tejiendo la red: el inventor del World Wide Web nos descubre su origen. // Madrid: Siglo Veintiuno. (2018-05-14).

Brobst, J. (2009). Evaluating the accessibility of Florida's public library home pages. // Libri. 59:2, 88-103

Caballero-Cortes, L.; Faba-Pérez, C.; Moya-Anegón, F. (2009). Evaluación comparativa de la accesibilidad de los espacios web de las bibliotecas universitarias españolas y norteamericanas. // Investigación bibliotecológica. 23:47, 45-66. http://www.scielo.org.mx/pdf/ib/v23n47/v23 n47a3.pdf (2018-06-15).

Carrasco de la Rica, S.; Faba-Pérez, C. (2017). La accesibilidad web de las bibliotecas nacionales en Europa y América. // Cuadernos de Documentación Multimedia. 28:1, 125. https://revistas.ucm.es/index.php/CDMU/article/view File/55248/50301. (2018-06-16).

Conway, V. (2011). Website accessibility in Western Australian public libraries. // Australian Library Journal. 60:2, 103-112.

Delmàs Ruiz, M.; López Borrull, A. (2015). Perfil profesional en las bibliotecas públicas: visión de los mismos bibliotecarios. // BiD: textos universitaris de biblioteconomía i documentació. 35 . http://bid.ub.edu/es/35/delmas.htm. (2018-02-10) 
España. Ley 13/1994, de 1 de junio, de Autonomía del Banco de España. // Boletín Oficial del Estado. 131 (3 de junio de 1994) 131, 17400-17408. https://www.boe.es/buscar/doc.php?id=BOE-A-1994-12553 (2018-02-25).

España. Ministerio de Educación, Cultura y Deporte. http://www.mecd.gob.es/cultura/areas/bibliotecas/mc/ebp/presentacion.html (2018-07-23).

Fernández Cortés, J. M. (2009). Accesibilidad en banca online y cajeros automáticos: el caso de laCaixa. // No solo usabilidad journal. Julio. http://nosolousabilidad.com/articulos/accesibilidad lacaixa.htm (2019-02-02)

Ford, B. J. (2002). Todos son bienvenidos: la biblioteca pública como espacio de integración ciudadana. // I Congreso Nacional de Bibliotecas Públicas [en línea]. Valencia: España. Ministerio de Cultura, 190-199. http://travesia.mcu.es/portalnb/jspui/bitstream/10421/1150/1/Congr esoNacionalBP_01.pdf (2018-07-03).

Hassan Montero, Y.; Martín Fernández, F. J. (2003). Qué es la Accesibilidad Web. // No solo usabilidad journal, 2. http://www.nosolousabilidad.com/articulos/accesibilidad.htm. (2018-02-04).

Henry, S. L. (2002). Understanding web accessibility. // Web Accessibility: Web Standards and Regulatory Compliance. http://www.adobe.com/macromedia/accessibility/pub/acc sites chap01.pdf. (2018-02-15).

Hernández Ortega, B.; Jiménez, J.; Martín, M. J. (2010). Efecto de la calidad de un sitio web sobre el tráfico recibido: El caso de la banca electrónica en España. // Information research. 15:2. http://www.informationr.net/ir/152/paper429.html (2018-06-16)

IFLA (1994). Manifiesto de la IFLA/UNESCO sobre la Biblioteca Pública. https://www.ifla.org/node/7271 (2019-0604).

IFLA (2001). Directrices IFLA/UNESCO para el desarrollo del servicio de bibliotecas públicas [en línea]. Madrid: Federación Internacional de Asociaciones de Bibliotecarios y Bibliotecas, 2001. https://www.ifla.org/files/assets/hq/publications/archive/the-public-library-service/pg01-s.pdf (2018-02-05)

IFLA (2002). Statement on Libraries and Sustainable Development. https://www. ifla.org/publications/statement-on-libraries-and-sustainable-development (2019-06-04).

Khan, M. S.; Mahapatra, S. S.; Sreekumar. (2009). Service quality evaluation in internet banking: an empirical study in India. // International Journal of Indian Culture and Business Management. 2:1, 30-46. http://dspace.nitrkl.ac.in/dspace/bitstream/2080/746/1/sssm.pdf (201812-02).

Lara Guijarro, E. G.; Albán Silva, L. C. (2017). Los riesgos de las transacciones bancarias por Internet. // Revista Publicando. 4:10, 62-74. https://www.rmlconsultores.com/revista/index.php/crv/article/view/436/pdf_261 (2018-0701)

Lilly, E. B.; Van Fleet, C. (2000). Measuring the accessibility of public library home pages. // Reference and User Services Quarterly, 40:2, 156-163.

López López, P. (2007). Biblioteca y ética ciudadana. // Educación y Biblioteca [en línea], 159, (mayo/junio). http://eprints.ucm.es/9737/1/PedroLopez1.pdf (2018-07$02)$

López Marín, L.; Méndez Rodríguez, E.M.; Sorli Rojo, Á. (2002). Avaluació de l'accessibilitat i usabilitat dels llocs web de les biblioteques publiques catalanes. // Revista de biblioteconomía i documentació. 2008, 31. https://www. raco.cat/index.php/ltem/article/view/22572/22406 (201807-01).

López Miguens, M. J.; Rodríguez Comesaña, L.; González Vázquez, E. (2010). Importancia del sitio web en la banca online: influencia sobre la confianza. // Investigaciones
Europeas de Dirección y Economía de la Empresa. 16: 3 85-106. https://www.sciencedirect.com/science/article/pii/S1135252312600367. (2018-07-01).

Luján Mora, S. Accesibilidad web. / Legislación. http://accesibilidadweb.dlsi.ua.es/?menu=legislacion. (2018-04-05).

Maatta Smith, S. L. (2014). Web Accessibility Assessment of Urban Public Library Websites // Public Library Quarterly. 33:3, 187-204

Martínez, A. B.; Andrés, J. de; García, J. (2014). Determinants of the Web accessibility of European banks // Information Processing \& Management. 50:1, 69-86.

Merlo, I.; Picart, E.; Monforte, M. Help my cash. // Bancos online España - Tu banco 100\% digital. https://www.helpmycash.com/banco/bancos-online-espana/ (2018-0618)

Park, S. J. (2012). Measuring public library accessibility: A case study using GIS. // Library and Information Science Research. 34:1, 13-21

Peón Pérez, J. L. (1991). El reglamento de Bibliotecas Públicas del Estado y del Sistema Español de Bibliotecas. // Documentación de las Ciencias de la Información. 14, 8894. http://revistas.ucm.es/index.php/DCIN/article/view/ DCIN9191110085A/20245 (2018-02-24).

Pulido Granados, E.R.; Medina García, V. H. (2008). Modelo de medición y evaluación de la usabilidad en sitios web de la banca virtual en Colombia // Ingeniería y Universidad. 12 (Enero-Junio). https://www.redalyc.org/pdf/ 477/47712103.pdf (2019-01-20).

San Emeterio, J.; Retolaza, J. L. (2003). ¿Existe espacio para una banca ética? // Lan harremanak: Revista de relaciones laborales. 2, 127-163. http://base.socioeco.org/docs/5255-19425-1-pb.pdf (2018-07-02).

Sánchez García, S.; Yubero Jiménez, S. (2015). Función social de las bibliotecas públicas: nuevos espacios de aprendizaje y de inserción social. // El profesional de la información. 24:2, 103-112. https://recyt.fecyt.es//index.php/EPI/article/view/epi.2015.mar.03/18803 (201802-25).

Serrano Mascaraque, E. (2009). Herramientas para la evaluación de la accesibilidad web. // Documentación de las Ciencias de la Información. 32, 245-266. https://revistas.ucm.es/index.php/DCIN/article/viewFile/DCIN090911 0245A/18823 (2018-02-02)

\section{Apéndice}

BPE de España y bancos registrados en el BE. con establecimiento en el país (Fuentes: http://mapabpe.mcu.es/mapabpe.cmd y http://app.bde.es/ren/html/inicio.htm)

\section{Bibliotecas públicas del Estado}

Biblioteca Pública da Coruña Miguel González Garcés Biblioteca Pública del Estado de Albacete Biblioteca Pública José Martínez Ruíz, Azorín (Alicante)

Biblioteca Provincial de Almería

Biblioteca Pública de Ávila

Biblioteca Pública Bartolomé J. Gallardo (Badajoz)

Biblioteca Pública de Burgos

Biblioteca Pública A. Rodríguez-Moñino / M. Brey (Cáceres)

Biblioteca Provincial de Cádiz

Biblioteca Pública Castellón de la Plana

Biblioteca Pública del Estado en Ceuta

Biblioteca Pública del Estado en Ciudad Real

Biblioteca Provincial de Córdoba

Biblioteca Pública Fermín Caballero (Cuenca)

Biblioteca Pública del Estado en Gijón

Biblioteca Carles Rahola de Girona

Biblioteca Provincial de Granada

Biblioteca Pública del Estado de Guadalajara 
Biblioteca Provincial de Huelva

Biblioteca Pública del Estado en Huesca

Biblioteca Provincial de Jaén

Biblioteca Pública del Estado en Las Palmas

Biblioteca Pública de León

Biblioteca Pública de Lleida

Biblioteca de La Rioja

Biblioteca Pública de Lugo

Bibliotecas de la Comunidad de Madrid. No pasa TAW

Biblioteca Pública Maó

Biblioteca Provincial de Málaga

Biblioteca Pública de Melilla

Biblioteca Pública del Estado Jesús Delgado Valhondo (Mérida)

Bibliotecas Públicos Región de Murcia

Biblioteca Pública de Ourense

Biblioteca Pública del Estado de Orihuela

Biblioteca de Asturias

Biblioteca Pública de Palencia

Biblioteca Pública de Palma

Biblioteca Pública de Pontevedra Antonio Odriozola

Biblioteca Pública de Salamanca

Biblioteca Pública del Estado en Santa Cruz de Tenerife

Biblioteca Central Cantabria

Biblioteca Pública de Santiago Ánxel Casal

Biblioteca Pública de Segovia

Biblioteca Provincial de Sevilla

Biblioteca Pública de Soria

Biblioteca Pública de Tarragona

Biblioteca Pública del Estado Teruel

Biblioteca Pública de Toledo

Biblioteca Pública del Estado de Valencia

Biblioteca de Castilla y León

Biblioteca Pública de Vitoria-Gasteiz. No pasa TAW

Biblioteca Pública de Zamora

Biblioteca Pública del Estado en Zaragoza

\section{Bancos}

Abanca Corporación Bancaria, S.A.

A\&G Banca Privada, S.A.

Allfunds Bank, S.A.

Andbank España, S.A.

Aresbank, S.A.

Banca March, S.A.

Banca Pueyo, S.A.

Banco Alcalá, S.A.

Banco Bilbao Vizcaya Argentaria, S.A.

Banco Caixa Geral, S.A.

Banco Caminos, S.A.

Banco Cetelem, S.A.
Banco Cooperativo Español, S.A.

Banco de Albacete, S.A.

Banco de Caja España de Inversiones, Salamanca y Soria,

S.A. No dispone de web

Banco de Castilla-La Mancha, S.A. No dispone de web

Banco de Crédito Social Cooperativo, S.A.

Banco de Depósitos, S.A.

Banco de Sabadell, S.A.

Banco Europeo de Finanzas, S.A.

Banco Finantia Sofinloc, S.A.

Banco Industrial de Bilbao, S.A. No dispone de web

Banco Inversis, S.A.

Banco Mediolanum, S.A.

Banco Occidental, S.A.

Banco Pastor, S.A.

Banco Pichincha España, S.A.

Banco Popular Español, S.A.

Banco Santander, S.A.

Bancofar, S.A.

Bank Degroof Petercam Spain, S.A.

Bankia, S.A.

Bankinter, S.A

Bankoa, S.A.

Banque Marocaine du Commerce Exterieur International, S.A.

BnP Paribas España, S.A.

Caixabank, S.A.

Cajasur Banco, S.A.

Cecabank, S.A.

Citibank España, S.A. No dispone de web

Deutsche Bank, Sociedad anónima española

Ebn Banco de negocios, S.A.

Evo Banco S.A.

Ibercaja Banco, S.A.

Kutxabank, S.A.

Liberbank, S.A.

Nuevo Micro Bank, S.A.

Open Bank, S.A.

Popular Banca Privada, S.A.

Renta 4 Banco, S.A.

Sabadell Consumer Finance, S.A.

Santander Consumer Finance, S.A. Web no operativa

Santander Investment, S.A.

Santander Securities Services, S.A.

Self-Trade Bank, S.A. Web no operativa

Targobank, S.A.

Unicaja Banco, S.A.

Wizink Bank, S.A.

Enviado: 2019-04-01. Segunda versión: 2019-06-05 Aceptado: 2019-06-14 